\title{
Referees 2013
}

Through their evaluation of manuscripts, the referees contribute greatly to the scientific standard of our journal. As a sign of our appreciation of this unselfish activity, we have decided to publish a list of referees. The following referees reviewed papers that were published in volume 223:

\begin{tabular}{lll}
$\begin{array}{l}\text { Abzhanov A } \\
\text { Albertson C } \\
\text { Alvarez J }\end{array}$ & Jiao R & Ramamurthy B \\
Jockusch EL & Rebecchi L \\
Rhat K & Kawli T & \\
Blum M & Khurana JP & Satoh N \\
Braeunig P & Kozmik Z & Schlosser G \\
Brewer P & Kuo DH & Schmidt-Ott U \\
Brown S & Kwon C & Schneider I \\
Budd GE & & Scholtz G \\
& Li X & Schweickert A \\
Damen WGM & & Seaver E \\
Degnan B & Mahaffey J & Song MH \\
del Pino EM & McClay D & Steele R \\
& Menke D & Stollewerk A \\
Ekker M & Minelli A & Suh W \\
Erwin D & Minokawa T & Sureshkumar S \\
Fujiwara S & Szathmary E \\
Gakanishi N & Thompson E \\
Goll M & Nath U & Todesco M \\
Gontijo A & & Trueta C \\
Guo YL & Obbard D & \\
Harvey T & Onai T & Valles Y \\
Hassel M & & \\
Hidalgo A & Parsons X & Winchell C \\
Hüttenhofer A & Pera E & Woodard C \\
& Pick L & Yu JK \\
\hline
\end{tabular}

